Copyright (@ 2009 Institute of Electrical and electronics Engineers, Inc.

All Rights reserved.

Personal use of this material, including one hard copy reproduction, is permitted.

Permission to reprint, republish and/or distribute this material in whole or in part for any other purposes must be obtained from the IEEE.

For information on obtaining permission, send an e-mail message to stds-igr@ieee.org.

By choosing to view this document, you agree to all provisions of the copyright laws protecting it.

Individual documents posted on this site may carry slightly different copyright restrictions.

For specific document information, check the copyright notice at the beginning of each document. 


\title{
On Designing Fuzzy Controllers for a Class of Nonlinear Networked Control Systems
}

\author{
Xiefu Jiang and Qing-Long Han
}

\begin{abstract}
This paper is concerned with controller design for a class of nonlinear networked control systems. These systems are approximated by uncertain linear networked Takagi-Sugeno (T-S) models with both network-induced delay and data packet dropout. Sufficient conditions are derived for the existence of a fuzzy controllers. Then, an iterative algorithm for the controller design is proposed. A control problem of a flexible-joint robot arm system is studied to show the effectiveness of the iterative algorithm.
\end{abstract}

Index Terms-Controller design, data packet dropout, fuzzy, networked control systems, network-induced delay, nonlinear systems, Takagi-Sugeno (T-S) model.

\section{INTRODUCTION}

$\mathbf{T}$ HE TREND of modern manufacturing plants and distributed industrial processes/control systems is to integrate computing, communication, and control into different levels of factory operations and information processes. In traditional control systems, system components such as sensors, controllers, and actuators are connected together through point-to-point cables [1]. However, expanding physical setups and functionality are pushing the limits of the point-to-point architecture [2]. Recently, as a result of the vast progress in communication technology, as network technology becomes cheaper and more reliable than fixed point-to-point connections, even in smalllocalized systems, more and more control systems will operate over networks, especially for control systems with a large number of distributed sensors and actuators. Murray [3], for example, foresees sensor, actuator, diagnostic, and command and coordination signals all traveling over a network. Compared with point-to-point architectures, several advantages of the network architectures include: reduced system wiring, plug and play devices, increased system agility, and ease of system diagnosis and maintenance. Therefore, modeling, analysis, and control of

Manuscript received April 4, 2006; revised October 25, 2006 and February 6, 2007; accepted May 26, 2007. This work was supported in part by Central Queensland University under the Research Advancement Awards Scheme Project "Robust Fault Detection, Filtering, and Control for Uncertain Systems with Time-Varying Delay" (January 2006-December 2008) and in part by Foundation of People's Republic of China Project "Analysis and Synthesis of Nonlinear Networked Control Systems" under Grant 60774058, and in part by the Hangzhou Dianzi University, China, under the Research Start-Up Foundation Grant KYS041507020.

X. Jiang is with the School of Automation, Hangzhou Dianzi University, Hangzhou 310018, China, on leave from the School of Computing Sciences, Faculty of Business and Informatics, Central Queensland University, Rockhampton, QLD 4702, Australia (e-mail: jiangxf@hdu.edu.cn).

Q.-L. Han is with both the Centre for Intelligent and Networked Systems and the School of Computing Sciences, Faculty of Business and Informatics, Central Queensland University, Rockhampton, QLD 4702, Australia (e-mail: q.han@cqu.edu.au).

Color versions of one or more of the figures in this paper are available online at http://ieeexplore.ieee.org.

Digital Object Identifier 10.1109/TFUZZ.2008.917293 networked control systems (NCSs) have recently emerged as a topic of much interest to community. Researchers from a variety of disciplines have become interested in the existing potential and challenges of NCSs (see, for example, [1], [2], [4]-[7], and references therein).

Due to the introduction of the network, two of the major challenges in NCSs still to be fully addressed are the effects of both network-induced delay and data packet dropout on the system performance, since both of them can degrade the performance of control systems designed without considering them and even destabilize the systems. Therefore, the challenging question which remains to be answered is how to quantitatively evaluate the effects of network-induced delay and data packet dropout. The answer to this question will significantly enhance the applications of NCSs in many manufacturing plants and distributed industrial processes/control systems, resulting in improved reliability, efficiency, and productivity. Some methodologies have been formulated based on several types of network behaviors and configurations in conjunction with different ways to treat the delay problem. For example, Halevi and Ray [8] proposed a methodology as the augmented deterministic discrete-time model to control a linear plant over a periodic delay network. Nilsson [9] proposed the optimal stochastic control method to control an NCS on random delay networks. Walsh et al. [10] considered a linear continuous plant and a continuous controller. They introduced the notation of maximum allowable transfer interval (MATI), which supposes that successive sensor messages are separated by at most MATI seconds. Gökas [11] used a modified Padé approximation and considered the network delay as an uncertainty, and designed a networked controller in the frequency domain using the robust control theory. Zhang et al. [7] used a hybrid system technique to study the stability of an NCS under the network-induced delay. However, most of the aforementioned papers fall into the case of constant timedelay. Existing constant time-delay control methodologies [12], [13] may not be directly suitable for controlling a system over the network, since network delays are usually time-varying, especially on the Internet. Therefore, to handle time-varying network-induced delays in a closed-loop control system over a network, a more advanced methodology is required.

In terms of the data packet dropout issue in the NCSs, because of the uncertainties and noise in the communication channels, there exist unavoidable errors in the transmitted data packet or even loss. If this happens, the corrupted data packet is dropped and the receiver (controller or actuator) uses the data packet that it received most recently. In addition, data packet dropout may occur when one packet, say, a sampled value from the sensor, reaches the destination later than its successors. In such 
a situation, the old data packet is dropped, and its successive data packet is used instead. However, very limited work has been done in examining the data packet dropout effect on the NCSs. In [14], the performance of the system as measured by the gain was expressed as a function of packet loss. Zhang et al. [7] modeled the NCSs with data packet dropouts as asynchronous switched systems. Ling and Lemmon [15] proposed optimal dropout compensation.

From this brief review, one can see that most of the existing results are concerned with the analysis and synthesis of linear NCSs [5]-[7]. For nonlinear NCSs, little attention has been received due to the systems' complexity and only a few results are available. Walsh et al. [16] proposed a two-step design approach to use standard control methodologies and to choose the network protocol and bandwidth in order to ensure important closed-loop properties are preserved when a computer network is inserted into the feedback loop. Nešic and Teel [17] addressed the inputto-state stability and $L_{p}$ stability of nonlinear NCSs and did not consider how to design the controller. Based on this fact, some new methods and approaches should be developed for designing controllers for nonlinear NCSs, which motivates this paper.

In this paper, we will investigate the effects of networkinduced delay and data packet dropout for a class of nonlinear networked control systems. Different from the methodology proposed by Walsh et al. [16], we will employ the TakagiSugeno (T-S) model approach [18]. More specifically, the nonlinear networked control systems will be approximated by uncertain linear networked T-S models. Then, we will give a sufficient condition for the existence of a fuzzy controller under consideration of both network-induced delay and data packet dropout. Based on this condition, we will propose an iterative algorithm for obtaining the controller. Finally, the proposed method on the application example of the control of a flexiblejoint robot arm through a network is illustrated to show the effectiveness of the iterative algorithm.

Notation. $\mathbb{R}^{n}$ denotes the $n$-dimensional Euclidean space. $\mathbb{R}^{m \times n}$ is the set of all $m \times n$ real matrices. The notation $P>0(P \geq 0)$ means that $P$ is symmetric and positive definite (positive semidefinite). For symmetric matrices $P$ and $Q$, the notation $P>Q(P \geq Q)$ means that matrix $P-Q$ is positive definite (positive semidefinite). $I$ is an identity matrix of appropriate dimensions. $\lambda_{i}(A)$ is the $i$ th eigenvalue of a real matrix $A$. For a real symmetric matrix $P, \lambda_{\max }(P)$ [respectively, $\left.\lambda_{\min }(P)\right]$ denotes the maximum (respectively, minimum) eigenvalue of the matrix $P .\|\cdot\|$ stands for the Euclidean vector norm or the induced matrix 2-norm as appropriate. $\operatorname{diag}\{\cdots\}$ denotes the block-diagonal matrix.

\section{Problem Statement}

Consider the following nonlinear system

$$
\dot{x}(t)=f(x(t))+g(x(t)) u(t)
$$

where $x(t) \in \mathbb{R}^{n}$ is the state vector and $u(t) \in \mathbb{R}^{p}$ is the input vector. We assume that $f(x(t))$ and $g(x(t))$ are sufficiently smooth on a domain $\mathbb{D} \subset \mathbb{R}^{n}$, and $f(0)=0$, where "sufficiently smooth" means that all the partial derivatives are defined and continuous. The initial condition of the system (1) is given by

$$
x\left(t_{0}\right)=x_{0} .
$$

Throughout this paper, we assume that systems (1) and (2) are controlled through a network and the system state is available for feedback.

From [18], the nonlinear system (1) can be represented by some simple local linear dynamic systems with their linguistic description as

$$
\begin{aligned}
& \text { Plant Rule } R^{i} \text { : } \\
& \text { IF } z_{1}(t) \text { is } M_{i 1}, z_{2}(t) \text { is } M_{i 2}, \ldots, z_{g}(t) \text { is } M_{i g} \\
& \text { THEN } \dot{x}(t)=A_{i} x(t)+B_{i} u(t)
\end{aligned}
$$

where $i=1,2, \ldots, r$ and $r$ is the number of IF-THEN rules; $z_{1}(t), z_{2}(t), \ldots, z_{g}(t)$ are the premise variables of (3) and $M_{i j}(i=1,2, \ldots, r ; j=1,2, \ldots, g)$ are the fuzzy sets corresponding to $z_{j}(t)$ and the plant rules; and $A_{i}$ and $B_{i}$ are known parameter matrices of appropriate dimensions.

By using a center average defuzzifier, product inference, and a singleton fuzzifier, the global dynamics of the T-S fuzzy systems (3) are described by

$$
\dot{x}(t)=\sum_{i=1}^{r} h_{i}(z(t))\left[A_{i} x(t)+B_{i} u(t)\right]
$$

where

$$
\begin{aligned}
h_{i}(z(t)) & =\frac{\mu_{i}(z(t))}{\sum_{i=1}^{r} \mu_{i}(z(t))}, \mu_{i}(z(t))=\prod_{j=1}^{g} M_{i j}\left(z_{j}(t)\right) \\
z(t) & =\left[z_{1}(t), z_{2}(t), \ldots, z_{g}(t)\right]
\end{aligned}
$$

in which $M_{i j}\left(z_{j}(t)\right)$ is the grade of membership of $z_{j}(t)$ in $M_{i j}$. Then, it can be seen that

$$
\mu_{i}(z(t)) \geq 0, i=1,2, \ldots, r, \sum_{i=1}^{r} \mu_{i}(z(t))>0, \forall t \geq t_{0} .
$$

Therefore

$$
h_{i}(z(t)) \geq 0, i=1,2, \ldots, r, \sum_{i=1}^{r} h_{i}(z(t))=1, \forall t \geq t_{0} .
$$

From (1) and (4), we have

$$
\begin{aligned}
\dot{x}(t)= & \sum_{i=1}^{r} h_{i}(z(t))\left[A_{i} x(t)+B_{i} u(t)\right] \\
& +\left(f(x(t))-\sum_{i=1}^{r} h_{i}(z(t)) A_{i} x(t)\right) \\
& +\left(g(x(t)) u(t)-\sum_{i=1}^{r} h_{i}(z(t)) B_{i} u(t)\right) \\
\triangleq & \sum_{i=1}^{r} h_{i}(z(t))\left[A_{i} x(t)+B_{i} u(t)\right]+\Delta f+\Delta g
\end{aligned}
$$

where $\Delta f+\Delta g$ is the approximation error between the nonlinear model (1) and the global fuzzy model (4). 


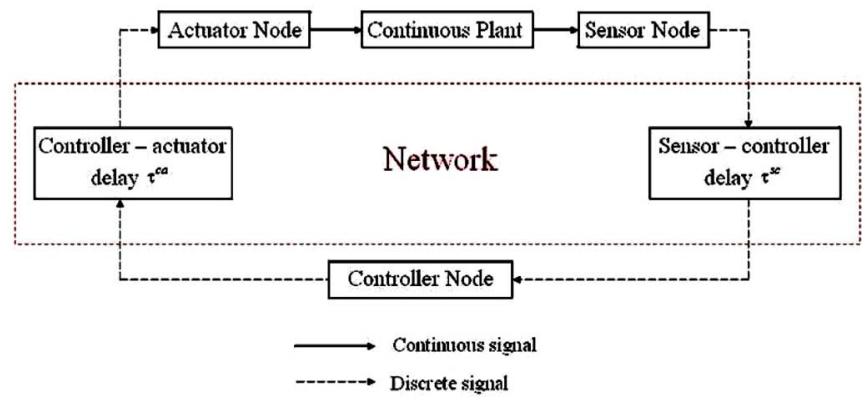

Fig. 1. Networked control system.

In the presence of the control network, which is shown in Fig. 1, data transfers between the controller and the remote system, e.g., sensors and actuators in a distributed control system, will induce networked delay in addition to the controller proceeding delay. As mentioned in the introduction, there are essentially three kinds of delay: (1) communication delay $\tau^{\mathrm{sc}}$ between the sensor and the controller; (2) computational delay $\tau^{c}$ in the controller; and (3) communication delay $\tau^{\text {ca }}$ between the controller and the actuator. Since the computational delay $\tau^{c}$ is usually very small, it is omitted in this paper.

First, since there exists the communication delay $\tau^{\text {sc }}$ between the sensor and the controller, which is shown in Fig. 1, the following state feedback T-S fuzzy-model-based control law is employed for the system (6) by utilizing the idea of parallel distributed compensation (PDC) [19], [20] in which the same fuzzy sets with the fuzzy model are shared for the designed fuzzy controller in the premise parts

$$
\begin{aligned}
R^{i}: & \operatorname{IF} z_{1}(t) \text { is } M_{i 1}, z_{2}(t) \text { is } M_{i 2}, \ldots, z_{g}(t) \text { is } M_{i g} \\
& \text { THEN } u\left(t^{+}\right)=K_{i} x\left(t-\tau_{k}^{s c}\right) \\
& t \in\left\{k h+\tau_{k}^{s c}, k=1,2, \ldots\right\}
\end{aligned}
$$

where $u\left(t^{+}\right)=\lim _{\xi \rightarrow t+0} u(\xi), K_{i}(i=1,2, \ldots, r)$ are the controller gains of (7) to be determined, and $h$ is the sampling period. Analogous to (4), the defuzzified output of the controller rules is given by

$$
\begin{aligned}
u\left(t^{+}\right) & =\sum_{i=1}^{r} h_{i}(z(t)) K_{i} x\left(t-\tau_{k}^{\mathrm{sc}}\right) \\
t & \in\left\{k h+\tau_{k}^{s c}, k=1,2, \ldots\right\} .
\end{aligned}
$$

In this paper, we assume that $u(t)=0$ before the first control signal reaches the plant. For the sake of simplicity, $h_{i}(z(t))$ is denoted by $h_{i}$ in the following.

Second, substituting (8) into (6) yields the closed-loop global fuzzy system of (6) by considering the communication delay $\tau^{\mathrm{ca}}$ between the controller and the actuator.

$$
\dot{x}(t)=\sum_{i=1}^{r} \sum_{j=1}^{r} h_{i} h_{j}\left[A_{i} x(t)+B_{i} K_{j} x(k h)\right]+\Delta f+\Delta g
$$

for $t \in\left[k h+\tau_{k},(k+1) h+\tau_{k+1}\right)$, where $\tau_{k}=\tau_{k}^{s c}+\tau_{k}^{c a}$, $k=1,2, \ldots, \Delta g=\sum_{i=1}^{r} \sum_{j=1}^{r} h_{i} h_{j}\left(g(x(t))-B_{i}\right) K_{j} x(k h)$. Considering the data packet dropout, the closed-loop global

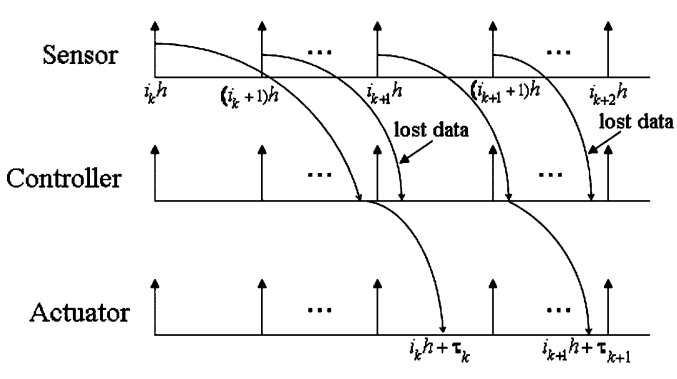

Fig. 2. Time diagram for data packets.

fuzzy system (9) can be modified as

$$
\dot{x}(t)=\sum_{i=1}^{r} \sum_{j=1}^{r} h_{i} h_{j}\left[A_{i} x(t)+B_{i} K_{j} x\left(i_{k} h\right)\right]+\Delta f+\Delta g
$$

where $\Delta g=\sum_{i=1}^{r} \sum_{j=1}^{r} h_{i} h_{j}\left(g(x(t))-B_{i}\right) K_{j} x\left(i_{k} h\right)$ for $t \in\left[i_{k} h+\tau_{k}, i_{k+1} h+\tau_{k+1}\right), i_{k}, k=1,2,3, \ldots$ are some integers, and $\left\{i_{1}, i_{2}, i_{3}, \ldots\right\} \subset\{0,1,2,3, \ldots\}$. The time-delay $\tau_{k}=\tau_{k}^{\mathrm{sc}}+\tau_{k}^{\mathrm{ca}}$ denotes the time from the instant $i_{k} h$ when sensor nodes sample sensor data from a plant to the instant when actuators transfer data to the plant (as shown in Fig. 2). Obviously, $\bigcup_{k=1}^{\infty}\left[i_{k} h+\tau_{k}, i_{k+1} h+\tau_{k+1}\right)=\left[t_{0}, \infty\right), t_{0} \geq 0$.

Suppose that there exist known real constant matrices $D_{i}, E_{i}$, and $E_{i b}(i=1,2, \ldots, r)$ of appropriate dimensions such that

$$
\Delta f=\sum_{i=1}^{r} h_{i} D_{i} F_{i}(t) E_{i} x(t)
$$

and

$$
\Delta g=\sum_{i=1}^{r} \sum_{j=1}^{r} h_{i} h_{j} D_{i} F_{i}(t) E_{i b} K_{j} x\left(i_{k} h\right)
$$

where $F_{i}(t) \in \mathbb{R}^{l \times m} \quad(i=1,2, \ldots, r)$ are unknown timevarying matrix functions with Lebesgue measurable elements satisfying

$$
F_{i}^{T}(t) F_{i}(t) \leq I, i=1,2, \ldots, r .
$$

Then, (10) can be rewritten as

$$
\dot{x}(t)=\sum_{i=1}^{r} \sum_{j=1}^{r} h_{i} h_{j}\left[A_{i}(t) x(t)+B_{i}(t) K_{j} x\left(i_{k} h\right)\right]
$$

for $t \in\left[i_{k} h+\tau_{k}, i_{k+1} h+\tau_{k+1}\right), k=1,2, \ldots$, where $A_{i}(t)=$ $A_{i}+\Delta A_{i}(t) \quad$ and $\quad B_{i}(t)=B_{i}+\Delta B_{i}(t) \quad(i=1,2, \ldots, r)$. $\Delta A_{i}(t)$ and $\Delta B_{i}(t)$ are unknown real matrices of appropriate dimensions representing time-varying parameter uncertainties of the system (14) and satisfying

$$
\left[\Delta A_{i}(t) \quad \Delta B_{i}(t)\right]=D_{i} F_{i}(t)\left[E_{i} \quad E_{i b}\right], i=1,2, \ldots, r .
$$

The following assumptions and definitions are required throughout the paper.

Assumption 1: [5], [6] The sensor is clock-driven; the controller and actuator are event-driven. 
Assumption 2: [5], [6] There exist two constants $\tau_{m} \geq 0$ and $\eta>0$ such that

$$
\left\{\begin{array}{l}
\left(i_{k+1}-i_{k}\right) h+\tau_{k+1} \leq \eta \\
\tau_{k} \geq \tau_{m}, k=1,2, \ldots
\end{array}\right.
$$

Remark 1: Since $x\left(i_{k} h\right)=x\left(t-\left(t-i_{k} h\right)\right)$, defining $\tau(t)=$ $t-i_{k} h, \quad t \in\left[i_{k} h+\tau_{k}, i_{k+1} h+\tau_{k+1}\right), k=1,2, \ldots, \quad$ we rewrite (14) as

$$
\dot{x}(t)=\sum_{i=1}^{r} \sum_{j=1}^{r} h_{i} h_{j}\left[A_{i}(t) x(t)+B_{i}(t) K_{j} x(t-\tau(t))\right]
$$

where $\tau(t)$ is piecewise-linear with derivative $\dot{\tau}(t)=1$ for $t \neq t_{k}$ and is discontinuous at the points $t=t_{k}, k=1,2, \ldots$. It is clear that $\tau_{k} \leq \tau(t) \leq\left(i_{k+1}-i_{k}\right) h+\tau_{k+1} \leq \eta$ for $t \in$ $\left[i_{k} h+\tau_{k}, i_{k+1} h+\tau_{k+1}\right), k=1,2, \ldots$ We supplement the initial condition of the state $x(t)$ on $\left[t_{0}-\eta, t_{0}\right]$ as

$$
x(t)=\phi(t), t \in\left[t_{0}-\eta, t_{0}\right]
$$

with $\phi\left(t_{0}\right)=x_{0}$, where $\phi(t)$ is a continuous function on $\left[t_{0}-\right.$ $\left.\eta, t_{0}\right]$.

Defnition 1: System (17) is said to be robustly exponentially stable if there exist constants $\alpha>0$ and $\beta>0$ such that $\|x(t)\| \leq \alpha \sup _{t_{0}-\eta \leq s \leq t_{0}}\|\phi(s)\| e^{-\beta\left(t-t_{0}\right)}$ for $t \geq t_{0}$ for all admissible uncertainties satisfying (15).

From Remark 1, it is clear to see that system (14) is equivalent to the delay system (17). In the following, system (14) being robustly exponentially stable means that the delay system (17) is robustly exponentially stable.

To end this section, we introduce the following lemmas which are useful in deriving the sufficient condition of the existence of fuzzy controllers.

Lemma 1: For two matrices $Z \in \mathbb{R}^{n \times m}, G \in \mathbb{R}^{n \times m}$, and a symmetric positive definite matrix $P \in \mathbb{R}^{n \times n}$, we have

$$
-G^{T} P^{-1} G \leq Z^{T} P Z-G^{T} Z-Z^{T} G .
$$

Equality holds if and only if $Z=P^{-1} G$.

Proof: It is easy to see that

$$
(P Z-G)^{T} P^{-1}(P Z-G) \geq 0 .
$$

Rearranging this matrix inequality yields (19). The equality holds if and only if $P Z=G$, i.e., $Z=P^{-1} G$.

Lemma 2: [21] For any constant matrix $W \in \mathbb{R}^{n \times n}, W=$ $W^{T}>0$, scalar $\gamma>0$, and vector function $\dot{x}:[-\gamma, 0] \rightarrow \mathbb{R}^{n}$ such that the following integration is well defined, then

$$
\begin{aligned}
& -\gamma \int_{-\gamma}^{0} \dot{x}^{T}(t+\xi) W \dot{x}(t+\xi) d \xi \\
& \quad \leq\left(\begin{array}{ll}
x^{T}(t) & x^{T}(t-\gamma)
\end{array}\right)\left(\begin{array}{cc}
-W & W \\
W & -W
\end{array}\right)\left(\begin{array}{c}
x(t) \\
x(t-\gamma)
\end{array}\right) .
\end{aligned}
$$

\section{MAIN RESULT}

Notice that the system described by (13)-(15) can be rewritten as [22], [23]

$$
\dot{x}(t)=\sum_{i=1}^{r} \sum_{j=1}^{r} h_{i} h_{j}\left[A_{i} x(t)+B_{i} K_{j} x\left(i_{k} h\right)+D_{i} v_{i j}(t)\right]
$$

subject to uncertain feedback

$$
\begin{aligned}
& v_{i j}(t)=F_{i}(t) g_{i j}(t), \\
& g_{i j}(t)=E_{i} x(t)+E_{i b} K_{j} x\left(i_{k} h\right)
\end{aligned}
$$

for $t \in\left[i_{k} h+\tau_{k}, i_{k+1} h+\tau_{k+1}\right), k=1,2, \ldots$ In view of (13), (21) and (22), we have

$$
\begin{aligned}
v_{i j}^{T}(t) v_{i j}(t) \leq\left[E_{i} x(t)+E_{i b}\right. & \left.K_{j} x\left(i_{k} h\right)\right]^{T} \\
& \times\left[E_{i} x(t)+E_{i b} K_{j} x\left(i_{k} h\right)\right] .
\end{aligned}
$$

The following proposition gives a sufficient condition of the existence of the fuzzy controller of system (14).

Proposition 1: For given scalars $\tau_{m}$ and $\eta$, system (14) is robustly exponentially stable for network-induced delay and data packet dropout satisfying (16) and all admissible uncertainties by (13) and (15), if there exist a scalar $\tilde{\varepsilon}>0$, some matrices $X>0, \tilde{Q}>0, R>0, S>0$, and $Y_{j}(j=1,2, \ldots, r)$ of appropriate dimensions such that

$$
\left\{\begin{array}{l}
\Xi_{i i}<0, \quad \text { for } 1 \leq i \leq r \\
\Xi_{i j}+\Xi_{j i}<0, \quad \text { for } 1 \leq i<j \leq r
\end{array}\right.
$$

simultaneously hold, where

$$
\Xi_{i j}=\left[\begin{array}{ccccccc}
\Gamma_{11} & \Gamma_{12} & \Gamma_{13} & \tilde{\varepsilon} D_{i} & \Gamma_{15} & \Gamma_{16} & \Gamma_{17} \\
\Gamma_{12}^{T} & \Gamma_{22} & 0 & 0 & \Gamma_{25} & \Gamma_{26} & \Gamma_{27} \\
\Gamma_{13}^{T} & 0 & \Gamma_{33} & 0 & \Gamma_{25} & \Gamma_{26} & \Gamma_{27} \\
\tilde{\varepsilon} D_{i}^{T} & 0 & 0 & -\tilde{\varepsilon} I & \Gamma_{45} & \Gamma_{46} & 0 \\
\Gamma_{15}^{T} & \Gamma_{25}^{T} & \Gamma_{25}^{T} & \Gamma_{45}^{T} & -R^{-1} & 0 & 0 \\
\Gamma_{16}^{T} & \Gamma_{26}^{T} & \Gamma_{26}^{T} & \Gamma_{46}^{T} & 0 & -S^{-1} & 0 \\
\Gamma_{17}^{T} & \Gamma_{27}^{T} & \Gamma_{27}^{T} & 0 & 0 & 0 & -\tilde{\varepsilon} I
\end{array}\right]
$$

with

$$
\begin{aligned}
\delta & =\eta-\tau_{m} \\
\Gamma_{11} & =A_{i} X+B_{i} Y_{j}+\left(A_{i} X+B_{i} Y_{j}\right)^{T} \\
\Gamma_{12} & =B_{i} Y_{j} \\
\Gamma_{13} & =-\tilde{Q}+B_{i} Y_{j} \\
\Gamma_{15} & =\tau_{m}\left(A_{i} X+B_{i} Y_{j}\right)^{T} \\
\Gamma_{16} & =\delta\left(A_{i} X+B_{i} Y_{j}\right)^{T} \\
\Gamma_{17} & =\left(E_{i} X+E_{i b} Y_{j}\right)^{T} \\
\Gamma_{22} & =-X S X \\
\Gamma_{25} & =\tau_{m}\left(B_{i} Y_{j}\right)^{T} \\
\Gamma_{26} & =\delta\left(B_{i} Y_{j}\right)^{T}
\end{aligned}
$$




$$
\begin{aligned}
& \Gamma_{27}=\left(E_{i b} Y_{j}\right)^{T} \\
& \Gamma_{33}=-\tilde{Q}-X R X \\
& \Gamma_{45}=\tau_{m} \tilde{\varepsilon} D_{i}^{T} \\
& \Gamma_{46}=\delta \tilde{\varepsilon} D_{i}^{T} .
\end{aligned}
$$

Moreover, the controller gains of (8) are given by $K_{j}=Y_{j} X^{-1}$ for $j=1,2, \ldots, r$.

Proof: See the Appendix.

Notice that (24) is not a linear matrix inequality (LMI), so it cannot be solved by MATLAB LMI Toolbox. Using Lemma 1, (24) is implied by

$$
\left\{\begin{array}{l}
\Phi(i, i)<0, \text { for } 1 \leq i \leq r \\
\Phi(i, j)+\Phi(j, i)<0, \text { for } 1 \leq i<j \leq r
\end{array}\right.
$$

where

$$
\Phi(i, j)=\left[\begin{array}{ccccccc}
\hat{\Gamma}_{11} & \hat{\Gamma}_{12} & \hat{\Gamma}_{13} & \tilde{\varepsilon} D_{i} & \hat{\Gamma}_{15} & \hat{\Gamma}_{16} & \hat{\Gamma}_{17} \\
\hat{\Gamma}_{12}^{T} & \hat{\Gamma}_{22} & 0 & 0 & \hat{\Gamma}_{25} & \hat{\Gamma}_{26} & \hat{\Gamma}_{27} \\
\hat{\Gamma}_{13}^{T} & 0 & \hat{\Gamma}_{33} & 0 & \hat{\Gamma}_{25} & \hat{\Gamma}_{26} & \hat{\Gamma}_{27} \\
\tilde{\varepsilon} D_{i}^{T} & 0 & 0 & -\tilde{\varepsilon} I & \hat{\Gamma}_{45} & \hat{\Gamma}_{46} & 0 \\
\hat{\Gamma}_{15}^{T} & \hat{\Gamma}_{25}^{T} & \hat{\Gamma}_{25}^{T} & \hat{\Gamma}_{45}^{T} & -\tilde{R} & 0 & 0 \\
\hat{\Gamma}_{16}^{T} & \hat{\Gamma}_{26}^{T} & \hat{\Gamma}_{26}^{T} & \hat{\Gamma}_{46}^{T} & 0 & -\tilde{S} & 0 \\
\hat{\Gamma}_{17}^{T} & \hat{\Gamma}_{27}^{T} & \hat{\Gamma}_{27}^{T} & 0 & 0 & 0 & -\tilde{\varepsilon} I
\end{array}\right]
$$

with

$$
\begin{aligned}
& \hat{\Gamma}_{11}=A_{i} X+B_{i} Y_{j}+\left(A_{i} X+B_{i} Y_{j}\right)^{T} \\
& \hat{\Gamma}_{12}=B_{i} Y_{j} \\
& \hat{\Gamma}_{13}=-\tilde{Q}+B_{i} Y_{j} \\
& \hat{\Gamma}_{15}=\tau_{m}\left(A_{i} X+B_{i} Y_{j}\right)^{T} \\
& \hat{\Gamma}_{16}=\delta\left(A_{i} X+B_{i} Y_{j}\right)^{T} \\
& \hat{\Gamma}_{17}=\left(E_{i} X+E_{i b} Y_{j}\right)^{T} \\
& \hat{\Gamma}_{22}=Z^{T} \tilde{S} Z-Z^{T} X-X Z \\
& \hat{\Gamma}_{25}=\tau_{m}\left(B_{i} Y_{j}\right)^{T} \\
& \hat{\Gamma}_{26}=\delta\left(B_{i} Y_{j}\right)^{T} \\
& \hat{\Gamma}_{27}=\left(E_{i b} Y_{j}\right)^{T} \\
& \hat{\Gamma}_{33}=\tilde{Z} \tilde{Z}^{T} \tilde{R}-\tilde{Z}^{T} X-X \tilde{Z}-\tilde{Q} \\
& \hat{\Gamma}_{45}=\tau_{m} \tilde{\varepsilon} D_{i}^{T} \\
& \hat{\Gamma}_{46}=\delta \tilde{\varepsilon} D_{i}^{T}
\end{aligned}
$$

for two given $n \times n$ matrices $Z$ and $\tilde{Z}$, where $\tilde{R}=R^{-1}, \tilde{S}=$ $S^{-1}$. (25) is equivalent to (24) if and only if $Z=\tilde{S}^{-1} X$ and $\tilde{Z}=\tilde{R}^{-1} X$.

One can clearly see that the nonlinear matrix inequality (24) is solvable for $\tau_{m}=\delta=0$ if (24) is solvable for $\tau_{m}>0$ and $\delta>0$. For $\tau_{m}=\delta=0$, by pre- and postmultiplying both sides of the nonlinear matrix inequality (24) with the diagonal matrix $\operatorname{diag}\{I, I, I, I, I, X R, X S, I, I\}$ and its transpose, respec- tively, one can obtain that (24) is equivalent to

$$
\left\{\begin{array}{l}
\tilde{\Phi}(i, i)<0, \quad \text { for } 1 \leq i \leq r \\
\tilde{\Phi}(i, j)+\tilde{\Phi}(j, i)<0, \quad \text { for } 1 \leq i<j \leq r
\end{array}\right.
$$

where

$$
\tilde{\Phi}(i, j)=\left[\begin{array}{ccccccc}
\Gamma_{11} & \Gamma_{12} & \Gamma_{13} & \tilde{\varepsilon} D_{i} & 0 & 0 & \Gamma_{17} \\
\Gamma_{12}^{T} & -\bar{S} & 0 & 0 & 0 & 0 & \Gamma_{27} \\
\Gamma_{13}^{T} & 0 & \bar{\Gamma}_{33} & 0 & 0 & 0 & \Gamma_{27} \\
\tilde{\varepsilon} D_{i}^{T} & 0 & 0 & -\tilde{\varepsilon} I & 0 & 0 & 0 \\
0 & 0 & 0 & 0 & -\bar{R} & 0 & 0 \\
0 & 0 & 0 & 0 & 0 & -\bar{S} & 0 \\
\Gamma_{17}^{T} & \Gamma_{27}^{T} & \Gamma_{27}^{T} & 0 & 0 & 0 & -\tilde{\varepsilon} I
\end{array}\right]
$$

with $\bar{\Gamma}_{33}=-\tilde{Q}-\bar{R}, \bar{R}=X R X$, and $\bar{S}=X S X$. If (26) has a feasible solution $\left(\tilde{\varepsilon}, X, \tilde{Q}, \bar{R}, \bar{S}, Y_{j}\right)$ for $j=1,2, \ldots, r$, then (25) is solvable with a feasible solution $(\tilde{\varepsilon}, X, \tilde{Q}, \tilde{R}=$ $\left.X \bar{R}^{-1} X, \tilde{S}=X \bar{S}^{-1} X, Y_{j}\right)$ for $j=1,2, \ldots, r, Z=X^{-1} \bar{S}$, $\tilde{Z}=X^{-1} \bar{R}$, and $\tau_{m}=\delta=0$. Therefore, there exist $\tau_{m}>0$ and $\delta>0$ such that (25) is solvable for $Z=X^{-1} \bar{S}$ and $\tilde{Z}=X^{-1} \bar{R}$. If (26) is not solvable, then the original nonlinear matrix inequality (24) is not solvable for any $\tau_{m}>0$ or $\delta>0$.

Based on the aforesaid discussion, one can see that two constant matrices $Z$ and $\tilde{Z}$ are introduced for solving nonlinear matrix inequality (24). As a result, they need to be given in advance for solving the LMI (25). In general, it is not easy to determine the matrices $Z$ and $\tilde{Z}$ in advance before solving the LMI (25). By Lemma 1, if there exist a scalar $\tilde{\varepsilon}>0$, some matrices $X>0, \tilde{Q}>0, \tilde{R}>0, \tilde{S}>0$, and $Y_{j}$ of appropriate dimensions such that (25) holds for two given $n \times n$ matrices $Z$ and $\tilde{Z}$, then (25) holds for $\tilde{\varepsilon}>0, X>0, \tilde{Q}>0, \tilde{R}>0, \tilde{S}>0$, $Y_{j}(j=1,2, \ldots, r$,$) and Z=\tilde{S}^{-1} X, \tilde{Z}=\tilde{R}^{-1} X$. Therefore, the following novel iterative algorithm is proposed for finding a maximum interval $\left[\tau_{m}^{*}, \tau_{m}^{*}+\delta\right]$ such that the closed-loop system (14) is robustly exponentially stable for any networkinduced delay and data packet dropout satisfying (16) by solving the nonlinear matrix inequality (24), and the matrices $Z$ and $\tilde{Z}$ do not need to be given in advance, where $\tau_{m}^{*} \geq 0$ is a given constant.

Algorithm 1: 1) For $\tau_{m}=\delta=0$, find a feasible solution to the LMI (26)

$$
\left(\tilde{\varepsilon}_{0}, X_{0}, \tilde{Q}_{0}, \bar{R}_{0}, \bar{S}_{0}, Y_{j 0}\right)
$$

where $j=1,2, \ldots, r$. Set $Z_{1}=X_{0}^{-1} \bar{S}_{0}, \tilde{Z}_{1}=X_{0}^{-1} \bar{R}_{0}$, and $k=1$. If there is none, exit.

2) For two $n \times n$ matrices $Z=Z_{1}, \tilde{Z}=\tilde{Z}_{1}$, and a sufficiently small constant $\tau_{m}>0$, find a maximum $\delta=\delta_{1}$ such that the LMI (25) is solvable with a feasible solution

$$
\left(\tilde{\varepsilon}_{1}, X_{1}, \tilde{Q}_{1}, \tilde{R}_{1}, \tilde{S}_{1}, Y_{j 1}\right)
$$

where $j=1,2, \ldots, r$. Set $Z_{2}=\tilde{S}_{1}^{-1} X_{2}, \tilde{Z}_{2}=\tilde{R}_{1}^{-1} X_{1}$, and $k=k+1$.

3) For the matrices $Z_{k}, \tilde{Z}_{k}$ and the given constant $\tau_{m}>0$, find a maximum $\delta=\delta_{k}$ such that the LMI (25) is solvable with 


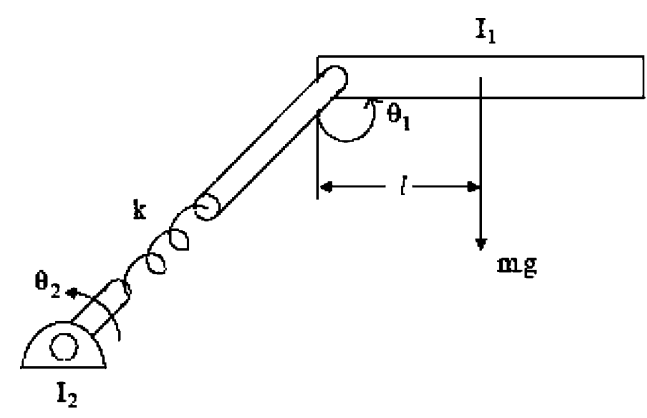

Fig. 3. Flexible-joint robot arm system.

a feasible solution for $j=1,2, \ldots, r$

$$
\left(\tilde{\varepsilon}_{k}, X_{k}, \tilde{Q}_{k}, \tilde{R}_{k}, \tilde{S}_{k}, Y_{j k}\right)
$$

4) If a stopping criterion is satisfied, exit. Otherwise, set $Z_{k+1}=\tilde{S}_{k}^{-1} X_{k}, \tilde{Z}_{k+1}=\tilde{R}_{k}^{-1} X_{k}$, and $k=k+1$. If $\tau_{m}<\tau_{m}^{*}$, then return to Step 3) after increasing $\tau_{m}$ to some extent until $\tau_{m}=\tau_{m}^{*}$. Otherwise, return to Step 3) directly.

Remark 2: Steps 1), 2) and every Step 3) of the algorithm are simple LMI problems, and they can be solved directly by using the MATLAB LMI Toolbox. The stopping criterion can be chosen as a specified number of iterations or the minimum increment of $\delta$.

The following proposition shows that the proposed algorithm is convergent.

Proposition 2: The sequence $\left\{\delta_{k}\right\}$ is monotone increasing and bounded upper by $\delta_{M}$ for a given $\tau_{m}>0$, where $\delta_{M}>0$ is determined by a given time-delay system. Thus, the sequence $\left\{\delta_{k}\right\}$ converges to some value $\delta_{\infty} \leq \delta_{M}$.

Proof: It is easy to see from Lemma 1 that (25) implies (24). For $k>0$, given constant $\tau_{m}>0$ and matrices $Z_{k}, \tilde{Z}_{k}$, if there exist $\tilde{\varepsilon}_{k}, X_{k}, \tilde{Q}_{k}, \tilde{R}_{k}, \tilde{S}_{k}, Y_{j k}$ such that (25) holds for $\delta_{k}$, then (24) also holds. So (25) holds for $Z_{k+1}=\tilde{S}_{k}^{-1} X_{k}, \tilde{Z}_{k+1}=$ $\tilde{R}_{k}^{-1} X_{k}, \tilde{\varepsilon}_{k}, X_{k}, \tilde{Q}_{k}, \tilde{R}_{k}, \tilde{S}_{k}, Y_{j k}$, and $\delta_{k}$. Thus, for matrices $Z_{k+1}, \tilde{Z}_{k+1}$, and the given constant $\tau_{m}>0$, we can find a maximum $\delta_{k+1} \geq \delta_{k}$ such that (25) is solvable with a feasible solution $\tilde{\varepsilon}_{k+1}, X_{k+1}, \tilde{Q}_{k+1}, \tilde{R}_{k+1}, \tilde{S}_{k+1}, Y_{j, k+1}$. Moreover, for a given time-delay system (1) and a given constant $\tau_{m}>0$, there exists $\delta_{M}>0$ such that $\delta_{k} \leq \delta_{M}$.

Remark 3: One can find from the aforesaid discussion that if the original nonlinear matrix inequality (24) is solvable for some $\tau_{m}^{*}>0$ and $\delta^{*}>0$, then a solution to (24) can be found for some $0<\tau_{m} \leq \tau_{m}^{*}$ and $0<\delta \leq \delta^{*}$ by the proposed iterative algorithm. If no solution can be found for any $\tau_{m} \geq 0$ or $\delta \geq 0$ by the proposed iterative algorithm, then the original nonlinear matrix inequality (24) is not solvable for any $\tau_{m} \geq 0$ or $\delta \geq 0$.

\section{NUMERICAL EXAMPLE}

To illustrate the iterative algorithm for fuzzy controller design, we study the following control problem of a flexible-joint robot arm system, shown in Fig. 3 [24], which is controlled through a network.
Taking into account uncertainties, the flexible-joint robot arm model is considered as [24]

$$
\left\{\begin{array}{l}
\left(I_{1}+\delta I_{1}\right) \ddot{\theta}_{1}+(m g l+\delta m) \sin \left(\theta_{1}\right)+(k+\delta k)\left(\theta_{1}-\theta_{2}\right)=0 \\
\left(I_{2}+\delta I_{2}\right) \ddot{\theta}_{2}+(b+\delta b) \dot{\theta}_{2}+(k+\delta k)\left(\theta_{2}-\theta_{1}\right)=u+\delta u
\end{array}\right.
$$

where

$$
\begin{aligned}
& \left|\delta I_{1}\right| \leq c \cdot I_{1},\left|\delta_{2}\right| \leq c \cdot I_{2},|\delta m| \leq c \cdot m g l \\
& |\delta k| \leq c \cdot k,|\delta u| \leq c \cdot u,|\delta b| \leq c \cdot b .
\end{aligned}
$$

Let $x_{1}=\theta_{1}, x_{2}=\dot{\theta}_{1}, x_{3}=\theta_{2}$, and $x_{4}=\dot{\theta}_{2}$. Then, the state equation of the system is

$$
\dot{x}(t)=\left[\begin{array}{cccc}
0 & 1 & 0 & 0 \\
\Upsilon_{1} & 0 & \Upsilon_{2} & 0 \\
0 & 0 & 0 & 1 \\
\Upsilon_{3} & 0 & -\Upsilon_{3} & \Upsilon_{4}
\end{array}\right] x(t)+\left[\begin{array}{c}
0 \\
0 \\
0 \\
\frac{u(t)+\delta u(t)}{I_{2}+\delta I_{2}}
\end{array}\right]
$$

where

$$
\begin{aligned}
\Upsilon_{1} & =-\frac{m g l+\delta m}{I_{1}+\delta I_{1}} \cdot \frac{\sin x_{1}(t)}{x_{1}(t)}-\frac{k+\delta k}{I_{1}+\delta I_{1}} \\
\Upsilon_{2} & =\frac{k+\delta k}{I_{1}+\delta I_{1}}, \Upsilon_{3}=\frac{k+\delta k}{I_{2}+\delta I_{2}}, \Upsilon_{4}=-\frac{b+\delta b}{I_{2}+\delta I_{2}} \\
x(t) & =\left[x_{1}(t), x_{2}(t), x_{3}(t), x_{4}(t)\right]^{T} .
\end{aligned}
$$

The system parameters are taken to be $m=0.01 \mathrm{~kg}, I_{1}=$ $I_{2}=1 \mathrm{kgm}^{2}, k=0.05 \mathrm{~N} . \mathrm{m} / \mathrm{rad}, l=1 \mathrm{~m}, b=0.007 \mathrm{~N} . \mathrm{ms} / \mathrm{rad}$, $c=10 \%$, and $g=9.81 \mathrm{~m} / \mathrm{s}^{2}$ for illustrative purposes [24], [25]. Then, the following T-S model is used to approximate the nonlinear system (28):

$$
\left\{\begin{aligned}
\text { Plant Rule } R^{1}: \text { IF } x_{1}(t) \text { is about } 0 & \\
\text { THEN } \dot{x}(t)= & \left(A_{1}+D_{1} F_{1} E_{1}\right) x(t) \\
& +\left(B_{1}+D_{1} F_{1} E_{1 b}\right) u(t)
\end{aligned}\right.
$$

Plant Rule $R^{2}$ : IF $x_{1}(t)$ is about $\pm \pi / 2$

$$
\begin{aligned}
\text { THEN } \dot{x}(t)= & \left(A_{2}+D_{2} F_{2} E_{2}\right) x(t) \\
& +\left(B_{2}+D_{2} F_{2} E_{2 b}\right) u(t)
\end{aligned}
$$

where

$$
\begin{aligned}
A_{1} & =\left[\begin{array}{cccc}
0 & 1 & 0 & 0 \\
-0.1481 & 0 & 0.05 & 0 \\
0 & 0 & 0 & 1 \\
0.05 & 0 & -0.05 & -0.007
\end{array}\right] \\
A_{2} & =\left[\begin{array}{cccc}
0 & 1 & 0 & 0 \\
-0.1125 & 0 & 0.05 & 0 \\
0 & 0 & 0 & 1 \\
0.05 & 0 & -0.05 & -0.007
\end{array}\right] \\
B_{1} & =B_{2}=\left[\begin{array}{llll}
0 & 0 & 0 & 1
\end{array}\right]^{T}
\end{aligned}
$$



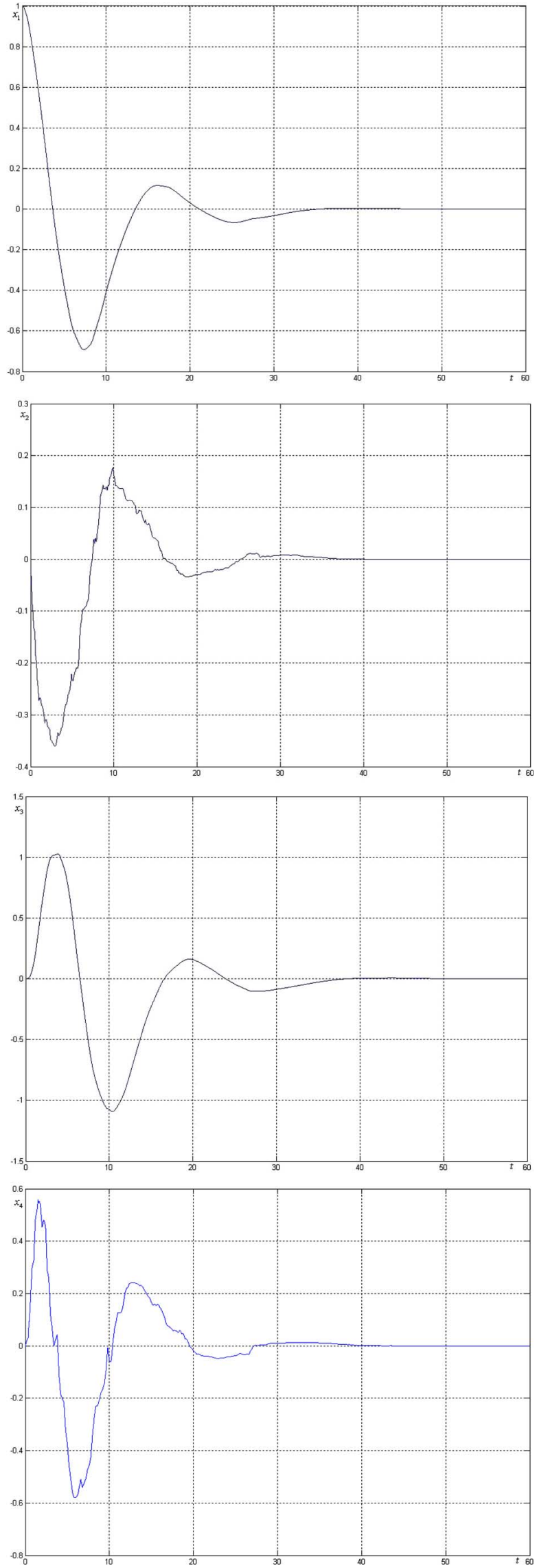

Fig. 4. Trajectories of states $x_{1}, x_{2}, x_{3}$, and $x_{4}$.

$$
\begin{aligned}
& D_{1}=D_{2}=\left[\begin{array}{llll}
1 & 0 & 0 & 0 \\
0 & 1 & 0 & 0 \\
0 & 0 & 1 & 0 \\
0 & 0 & 0 & 1
\end{array}\right] \\
& E_{1}=E_{2}=\left[\begin{array}{llll}
0 & 0 & 0 & 0 \\
\frac{2}{9} & 0 & \frac{2}{9} & 0 \\
0 & 0 & 0 & 0 \\
\frac{2}{9} & 0 & \frac{2}{9} & \frac{2}{9}
\end{array}\right], \quad E_{1 b}=E_{2 b}=\left[\begin{array}{l}
0 \\
0 \\
0 \\
\frac{2}{9}
\end{array}\right] .
\end{aligned}
$$

For this example, we assume that the nonlinear system (27) is controlled through a network, and $\tau_{m}=0.01 \mathrm{~s}$. By Algorithm 1, the closed-loop nonlinear system of the system (27) is exponentially stable for $\tau_{k} \in[0.01 s, 0.8441 s]$ under the fuzzy controller (7) with

$$
\begin{aligned}
K_{1} & =\left[\begin{array}{llll}
0.5272 & 0.0604 & -0.2735 & -0.8792
\end{array}\right] \\
K_{2} & =\left[\begin{array}{llll}
0.5333 & 0.0816 & -0.2696 & -0.8768
\end{array}\right] .
\end{aligned}
$$

The initial conditions are chosen as $x(0)=\left[\begin{array}{llll}1 & 0 & 0 & 0\end{array}\right]^{T}$. The sampling rate is chosen as $0.01 s$, that is, $h=0.01 s$. The simulation results for the four states of the nonlinear system are shown in Fig. 4, from which one can find that the system states are in equilibrium after $40 \mathrm{~s}$, while they need more than $2000 \mathrm{~s}$ from [25, Fig. 6]. Also, the peak value of Fig. 4 is much smaller than that of [25, Fig. 6].

\section{CONCLUSION}

We have addressed the problem of fuzzy controller design for a class of nonlinear networked control systems considering both time-varying network-induced delay and data packet dropout simultaneously. To the best of our knowledge, the problem has not been investigated in the open literature. We have employed uncertain networked Takagi-Sugeno models to approximate the nonlinear networked control systems. Then, we have derived some sufficient conditions for the existence of fuzzy controllers. Based on these conditions, we have proposed an iterative algorithm for achieving fuzzy controllers. In order to show the effectiveness of the iterative algorithm, we have studied the fuzzy control design of a flexible-joint robot arm through a network.

\section{APPENDIX}

\section{ProOF OF PROPOSITION 1}

Choose a functional as

$$
\begin{aligned}
V(t)= & x^{T}(t) P x(t)+\int_{t-\tau_{m}}^{t} x^{T}(s) Q x(s) d s \\
& +\tau_{m} \int_{-\tau_{m}}^{0} d s \int_{t+s}^{t} \dot{x}^{T}(\theta) R \dot{x}(\theta) d \theta \\
& +\delta \int_{-\eta}^{-\tau_{m}} d s \int_{t+s}^{t} \dot{x}^{T}(\theta) S \dot{x}(\theta) d \theta
\end{aligned}
$$

where $P>0, Q>0, R>0$, and $S>0$. 
Taking the derivative of $V(t)$ with respect to $t$ along the trajectory of (20) yields

$$
\begin{aligned}
\dot{V}(t)= & \sum_{i=1}^{r} \sum_{j=1}^{r} h_{i} h_{j} 2 x^{T}(t) P\left[A_{i} x(t)+B_{i} K_{j} x\left(i_{k} h\right)+D_{i} v_{i j}(t)\right] \\
& -x^{T}\left(t-\tau_{m}\right) Q x\left(t-\tau_{m}\right)+x^{T}(t) Q x(t) \\
& +\dot{x}^{T}(t)\left(\tau_{m}^{2} R+\delta^{2} S\right) \dot{x}(t)-\tau_{m} \int_{t-\tau_{m}}^{t} \dot{x}^{T}(s) R \dot{x}(s) d s \\
& -\delta \int_{t-\eta}^{t-\tau_{m}} \dot{x}^{T}(\theta) S \dot{x}(\theta) d \theta
\end{aligned}
$$

for $t \in\left[i_{k} h+\tau_{k}, i_{k+1} h+\tau_{k+1}\right), k=1,2, \ldots$

We use Lemma 2 to obtain

$$
\begin{aligned}
&-\tau_{m} \int_{t-\tau_{m}}^{t} \dot{x}^{T}(s) R \dot{x}(s) d s \\
& \quad \leq-\left[x(t)-x\left(t-\tau_{m}\right)\right]^{T} R\left[x(t)-x\left(t-\tau_{m}\right)\right]
\end{aligned}
$$

and

$$
\begin{aligned}
& -\delta \int_{t-\eta}^{t-\tau_{m}} \dot{x}^{T}(\theta) S \dot{x}(\theta) d \theta \leq-\delta \int_{i_{k} h}^{t-\tau_{m}} \dot{x}^{T}(\theta) S \dot{x}(\theta) d \theta \\
& \quad \leq-\left[x\left(t-\tau_{m}\right)-x\left(i_{k} h\right)\right]^{T} S\left[x\left(t-\tau_{m}\right)-x\left(i_{k} h\right)\right]
\end{aligned}
$$

From (31)-(33), we have

$$
\dot{V}(t) \leq \sum_{i=1}^{r} \sum_{j=1}^{r} h_{i} h_{j} \Upsilon_{i j}+\dot{x}^{T}(t) \Theta \dot{x}(t)
$$

for $t \in\left[i_{k} h+\tau_{k}, i_{k+1} h+\tau_{k+1}\right), k=1,2, \ldots$, where

$$
\begin{aligned}
\Theta= & \tau_{m}^{2} R+\delta^{2} S \\
\Upsilon_{i j}= & 2 x^{T}(t) P\left[A_{i} x(t)+B_{i} K_{j} x\left(i_{k} h\right)+D_{i} v_{i j}(t)\right] \\
& +x^{T}(t) Q x(t)-x^{T}\left(t-\tau_{m}\right) Q x\left(t-\tau_{m}\right) \\
& -\left[x(t)-x\left(t-\tau_{m}\right)\right]^{T} R\left[x(t)-x\left(t-\tau_{m}\right)\right] \\
& -\left[x\left(t-\tau_{m}\right)-x\left(i_{k} h\right)\right]^{T} S\left[x\left(t-\tau_{m}\right)-x\left(i_{k} h\right)\right] .
\end{aligned}
$$

By the Schur complement, it is easy to see that the following inequality implies that the right of (34) is a negative definite function.

$$
\left[\begin{array}{cc}
\sum_{i=1}^{r} \sum_{j=1}^{r} h_{i} h_{j} \Upsilon_{i j} & \dot{x}^{T}(t) \\
\dot{x}(t) & -\Theta^{-1}
\end{array}\right]<0 .
$$

From (20), the aforesaid inequality is written as

$$
\sum_{i=1}^{r} \sum_{j=1}^{r} h_{i} h_{j}\left[\begin{array}{cc}
\Upsilon_{i j} & \Gamma \\
\Gamma^{T} & -\Theta^{-1}
\end{array}\right]<0
$$

where $\Gamma=\left[A_{i} x(t)+B_{i} K_{j} x\left(i_{k} h\right)+D_{i} v_{i j}(t)\right]^{T}$. It is clear that (35) is implied by

$$
\left[\begin{array}{cc}
\Upsilon_{i j} & \Gamma \\
\Gamma^{T} & -\Theta^{-1}
\end{array}\right]<0
$$

for $i, j=1,2, \ldots, r$. We use the Schur complement to obtain

$$
\Upsilon_{i j}+\Gamma \Theta \Gamma^{T}<0
$$

which can be further written as

$$
\xi^{T} \Omega_{i j} \xi<0
$$

for $t \in\left[i_{k} h+\tau_{k}, i_{k+1} h+\tau_{k+1}\right), i, j=1,2, \ldots, r, k=1,2$, $\ldots$, where

$$
\begin{aligned}
\xi^{T} & =\left[\begin{array}{cccc}
x^{T}(t) & x^{T}\left(i_{k} h\right) & x^{T}\left(t-\tau_{m}\right) & v_{i j}^{T}(t)
\end{array}\right] \\
\Omega_{i j} & =\left[\begin{array}{cccc}
(1,1) & (1,2) & R & (1,4) \\
(1,2)^{T} & (2,2) & S & (2,4) \\
R & S & (3,3) & 0 \\
(1,4)^{T} & (2,4)^{T} & 0 & D_{i}^{T} \Theta D_{i}
\end{array}\right]
\end{aligned}
$$

with

$$
\begin{aligned}
& (1,1)=P A_{i}+A_{i}^{T} P+Q-R+A_{i}^{T} \Theta A_{i} \\
& (1,2)=P B_{i} K_{j}+A_{i}^{T} \Theta B_{i} K_{j} \\
& (1,4)=P D_{i}+A_{i}^{T} \Theta D_{i} \\
& (2,2)=-S+\left(B_{i} K_{j}\right)^{T} \Theta B_{i} K_{j} \\
& (2,4)=\left(B_{i} K_{j}\right)^{T} \Theta D_{i} \\
& (3,3)=-Q-R-S .
\end{aligned}
$$

Note that (23), by $\mathcal{S}$-procedure, (36) is implied by

$$
\tilde{\Xi}_{i j}+\tilde{\Xi}_{j i}<0
$$

for $\varepsilon>0$ and $1 \leq i \leq j \leq r$, where

$$
\tilde{\Xi}_{i j}=\Omega_{i j}+\varepsilon\left[\begin{array}{cccc}
E_{i}^{T} E_{i} & E_{i}^{T} E_{i b} K_{j} & 0 & 0 \\
\left(E_{i b} K_{j}\right)^{T} E_{i} & \left(E_{i b} K_{j}\right)^{T} E_{i b} K_{j} & 0 & 0 \\
0 & 0 & 0 & 0 \\
0 & 0 & 0 & -I
\end{array}\right] .
$$

It follows that there exists a $\lambda>0$ such that

$$
\dot{V}(t)<-\lambda x^{T}(t) x(t)-\lambda x^{T}\left(i_{k} h\right) x\left(i_{k} h\right)
$$

for $t \in\left[i_{k} h+\tau_{k}, i_{k+1} h+\tau_{k+1}\right), k=1,2, \ldots$ Next, we prove the robust exponential stability of the system (14) if the matrix inequality (37) holds. Define a new functional as

$$
\tilde{V}(t)=e^{\sigma t} V(t)
$$

where $\sigma>0$ is a constant to be determined. Then, using a similar method in [26], we obtain

$$
\begin{aligned}
\dot{\tilde{V}}(t)= & e^{\sigma t} \dot{V}(t)+\sigma e^{\sigma t} V(t) \\
\leq & -\lambda e^{\sigma t}\left\{x^{T}(t) x(t)+x^{T}\left(i_{k} h\right) x\left(i_{k} h\right)\right\}+\sigma e^{\sigma t} V(t) \\
\leq & \varphi_{1}(t)+\tau_{m} \sigma e^{\sigma t} \int_{-\tau_{m}}^{0} d s \int_{t+s}^{t} \dot{x}^{T}(\theta) R \dot{x}(\theta) d \theta \\
& +\delta \sigma e^{\sigma t} \int_{-\eta}^{-\tau_{m}} d s \int_{t+s}^{t} \dot{x}^{T}(\theta) S \dot{x}(\theta) d \theta
\end{aligned}
$$




$$
\begin{aligned}
= & \varphi_{1}(t)+\tau_{m} \sigma e^{\sigma t} \int_{t-\tau_{m}}^{t}\left(\theta-t+\tau_{m}\right) \dot{x}^{T}(\theta) R \dot{x}(\theta) d \theta \\
& +\delta \sigma e^{\sigma t} \int_{t-\eta}^{t-\tau_{m}}(\theta-t+\eta) \dot{x}^{T}(\theta) S \dot{x}(\theta) d \theta \\
& +\delta^{2} \sigma e^{\sigma t} \int_{t-\tau_{m}}^{t} \dot{x}^{T}(\theta) S \dot{x}(\theta) d \theta \\
\leq & \varphi_{1}(t)+\tau_{m}^{2} \sigma e^{\sigma t} \int_{t-\tau_{m}}^{t} \dot{x}^{T}(\theta) R \dot{x}(\theta) d \theta \\
& +\delta^{2} \sigma e^{\sigma t} \int_{t-\eta}^{t} \dot{x}^{T}(\theta) S \dot{x}(\theta) d \theta
\end{aligned}
$$

where $\varphi_{1}(t)=-\lambda e^{\sigma t}\left\{x^{T}(t) x(t)+x^{T}\left(i_{k} h\right) x\left(i_{k} h\right)\right\}+\sigma e^{\sigma t}$ $x^{T}(t) P x(t)+\sigma e^{\sigma t} \int_{t-\tau_{m}}^{t} x^{T}(s) Q x(s) d s$. Integrating both sides from $i_{k} h+\tau_{k}$ to $t$, we have

$$
\begin{aligned}
\tilde{V}(t) & -\tilde{V}\left(i_{k} h+\tau_{k}\right) \\
\leq & -\lambda \int_{i_{k} h+\tau_{k}}^{t} e^{\sigma s}\left\{x^{T}(s) x(s)+x^{T}\left(i_{k} h\right) x\left(i_{k} h\right)\right\} d s \\
& +\int_{i_{k} h+\tau_{k}}^{t} \sigma e^{\sigma s} x^{T}(s) P x(s) d s \\
& +\int_{i_{k} h+\tau_{k}}^{t} d s \int_{s-\tau_{m}}^{s} \sigma e^{\sigma s} x^{T}(\theta) Q x(\theta) d \theta \\
& +\tau_{m}^{2} \int_{i_{k} h+\tau_{k}}^{t} d s \int_{s-\tau_{m}}^{s} \sigma e^{\sigma s} \dot{x}^{T}(\theta) R \dot{x}(\theta) d \theta \\
& +\delta^{2} \int_{i_{k} h+\tau_{k}}^{t} d s \int_{s-\eta}^{s} \sigma e^{\sigma s} \dot{x}^{T}(\theta) S \dot{x}(\theta) d \theta .
\end{aligned}
$$

Since $V\left(x_{t}\right)$ is continuous on $\left[t_{0}, \infty\right)$, then from (38), it is easy to obtain

$$
\begin{aligned}
\tilde{V}(t) & -\tilde{V}\left(t_{0}\right) \\
\leq & \varphi_{2}(t)+\sigma \lambda_{\max }(Q) \int_{t_{0}}^{t} d s \int_{s-\tau_{m}}^{s} e^{\sigma s}\|x(\theta)\|^{2} d \theta \\
& +\sigma \tau_{m}^{2} \lambda_{\max }(R) \int_{t_{0}}^{t} d s \int_{s-\tau_{m}}^{s} e^{\sigma s}\|\dot{x}(\theta)\|^{2} d \theta \\
& +\sigma \delta^{2} \lambda_{\max }(S) \int_{t_{0}}^{t} d s \int_{s-\eta}^{s} e^{\sigma s}\|\dot{x}(\theta)\|^{2} d \theta \\
\leq & \varphi_{2}(t)+\sigma \lambda_{\max }(Q) \int_{t_{0}-\tau_{m}}^{t} d \theta \int_{\theta}^{\theta+\tau_{m}} e^{\sigma s}\|x(\theta)\|^{2} d s \\
& +\sigma \tau_{m}^{2} \lambda_{\max }(R) \int_{t_{0}-\tau_{m}}^{t} d \theta \int_{\theta}^{\theta+\tau_{m}} e^{\sigma s}\|\dot{x}(\theta)\|^{2} d s \\
& +\sigma \delta^{2} \lambda_{\max }(S) \int_{t_{0}-\eta}^{t} d \theta \int_{\theta}^{\theta+\eta} e^{\sigma s}\|\dot{x}(\theta)\|^{2} d s \\
\leq & \varphi_{2}(t)+\sigma \tau_{m} \lambda_{\max }(Q) e^{\sigma \tau_{m}} \int_{t_{0}-\tau_{m}}^{t} e^{\sigma \theta}|x(\theta)|^{2} d \theta \\
& +\sigma \tau_{m}^{3} \lambda_{\max }(R) e^{\sigma \tau_{m}} \int_{t_{0}-\tau_{m}}^{t} e^{\sigma \theta}\|\dot{x}(\theta)\|^{2} d \theta
\end{aligned}
$$

$$
\begin{aligned}
& +\sigma \delta^{2} \eta \lambda_{\max }(S) e^{\sigma \eta} \int_{t_{0}-\eta}^{t} e^{\sigma \theta}\|\dot{x}(\theta)\|^{2} d \theta \\
\leq & \varphi_{2}(t)+\sigma \tau_{m} \lambda_{\max }(Q) e^{\sigma \tau_{m}} \int_{t_{0}-\tau_{m}}^{t} e^{\sigma s}\|x(s)\|^{2} d s \\
& +\sigma \tau_{m}^{3} \lambda_{\max }(R) e^{\sigma \tau_{m}} \alpha_{1} \int_{t_{0}-\tau_{m}}^{t} e^{\sigma s}\|x(s)\|^{2} d s \\
& +\sigma \delta^{2} \eta \lambda_{\max }(S) e^{\sigma \eta} \alpha_{1} \int_{t_{0}-\eta}^{t} e^{\sigma s}\|x(s)\|^{2} d s \\
& +\sigma\left(\tau_{m}^{3} \lambda_{\max }(R) e^{\sigma \tau_{m}}+\delta^{2} \eta \lambda_{\max }(S) e^{\sigma \eta}\right) \alpha_{2} \varphi(t) \\
= & N_{1} \int_{t_{0}}^{t} e^{\sigma s}\|x(s)\|^{2} d s \\
& +\left(\sigma \tau_{m} \lambda_{\max }(Q) e^{\sigma \tau_{m}}\right. \\
& \left.+\sigma \tau_{m}^{3} \lambda_{\max }(R) e^{\sigma \tau_{m}} \alpha_{1}\right) \int_{t_{0}-\tau_{m}}^{t_{0}} e^{\sigma s}\|\phi(s)\|^{2} d s \\
& +\sigma \delta^{2} \eta \lambda_{\max }(S) e^{\sigma \eta} \alpha_{1} \int_{t_{0}-\eta}^{t_{0}} e^{\sigma s}\|\phi(s)\|^{2} d s+N_{2} \varphi(t)
\end{aligned}
$$

where

$$
\begin{aligned}
\varphi_{2}(t)= & -\lambda \int_{t_{0}}^{t} e^{\sigma s}\|x(s)\|^{2} d s-\lambda \varphi(t)+\sigma \lambda_{\max }(P) \\
& \times \int_{t_{0}}^{t} e^{\sigma s}\|x(s)\|^{2} d s \\
\varphi(t)= & \sum_{\iota=1}^{k-1} \int_{i_{\iota} h+\tau_{\iota}}^{i_{\iota} h+\tau_{\iota}+1} e^{\sigma s}\left\|x\left(i_{\iota} h\right)\right\|^{2} d s \\
& +\int_{i_{k} h+\tau_{k}}^{t} e^{\sigma s}\left\|x\left(i_{k} h\right)\right\|^{2} d s \\
\alpha_{1}= & \sum_{i=1}^{r}\left(\left\|A_{i}\right\|+\left\|D_{i}\right\| \cdot\left\|E_{i}\right\|\right) \\
\alpha_{2}= & \sum_{i, j=1}^{r}\left(\left\|B_{i}\right\|+\left\|D_{i}\right\| \cdot\left\|E_{i b}\right\|\right)\left\|K_{j}\right\| \\
N_{1}= & -\lambda+\sigma \lambda_{\max }(P)+\sigma \tau_{m} \lambda_{\max }(Q) e^{\sigma \tau_{m}} \\
& +\sigma\left(\tau_{m}^{3} \lambda_{\max }(R) e^{\sigma \tau_{m}}+\delta^{2} \eta \lambda_{\max }(S) e^{\sigma \eta}\right) \alpha_{1} \\
N_{2}= & -\lambda+\sigma\left(\tau_{m}^{3} \lambda_{\max }(R) e^{\sigma \tau_{m}}+\delta^{2} \eta \lambda_{\max }(S) e^{\sigma \eta}\right) \alpha_{2} .
\end{aligned}
$$

It is clear to see that there exists a sufficiently small constant $\sigma>0$ such that $N_{1}<0$ and $N_{2}<0$. Then, from (39), we have

$$
\begin{aligned}
\tilde{V}(t) \leq & \left(\sigma \tau_{m} \lambda_{\max }(Q) e^{\sigma \tau_{m}}+\sigma \tau_{m}^{3} \lambda_{\max }(R) e^{\sigma \tau_{m}} \alpha_{1}\right) \\
& \times \int_{t_{0}-\tau_{m}}^{t_{0}} e^{\sigma s}\|\phi(s)\|^{2} d s \\
& +\sigma \delta^{2} \eta \lambda_{\max }(S) e^{\sigma \eta} \alpha_{1} \int_{t_{0}-\eta}^{t_{0}} e^{\sigma s}\|\phi(s)\|^{2} d s \\
& +e^{\sigma t_{0}}\left(x_{0}^{T} P x_{0}+\int_{t_{0}-\tau_{m}}^{t_{0}} \phi^{T}(s) Q \phi(s) d s\right.
\end{aligned}
$$




$$
\begin{aligned}
& +\tau_{m} \lambda_{\max }(R) \alpha_{1} \int_{-\tau_{m}}^{0} d s \int_{t_{0}+s}^{t_{0}}\|\phi(\theta)\|^{2} d \theta \\
& \left.+\delta \lambda_{\max }(S) \alpha_{1} \int_{-\eta}^{-\tau_{m}} d s \int_{t_{0}+s}^{t_{0}}\|\phi(\theta)\|^{2} d \theta\right) .
\end{aligned}
$$

In consequence,

$$
\|x(t)\|^{2} \leq \frac{M_{1}+M_{2}}{\lambda_{\min }(P)} \sup _{t_{0}-\eta \leq s \leq t_{0}}\|\phi(\theta)\|^{2} e^{-\sigma\left(t-t_{0}\right)}
$$

where

$$
\begin{aligned}
M_{1}= & \tau_{m} \lambda_{\max }(Q) e^{\sigma \tau_{m}}+\tau_{m}^{3} \lambda_{\max }(R) e^{\sigma \tau_{m}} \alpha_{1} \\
& +\delta^{2} \eta \lambda_{\max }(S) e^{\sigma \eta} \alpha_{1} \\
M_{2}= & \lambda_{\max }(P)+\tau_{m} \lambda_{\max }(Q)+\frac{1}{2} \tau_{m}^{3} \lambda_{\max }(R) \alpha_{1} \\
& +\frac{1}{2} \delta^{2}\left(\eta+\tau_{m}\right) \lambda_{\max }(S) \alpha_{1} .
\end{aligned}
$$

So the system (14) is robustly exponentially stable if matrix inequality (37) holds. By the Schur complement, matrix inequality (37) is equivalent to

$$
\hat{\Xi}_{i j}+\hat{\Xi}_{j i}<0
$$

where

$$
\hat{\Xi}_{i j}=\left[\begin{array}{ccccccc}
\tilde{\Gamma}_{11} & \tilde{\Gamma}_{12} & R & P D_{i} & \tau_{m} A_{i}^{T} & \delta A_{i}^{T} & E_{i}^{T} \\
\tilde{\Gamma}_{12} & -S & S & 0 & \tilde{\Gamma}_{25} & \tilde{\Gamma}_{26} & \tilde{\Gamma}_{27} \\
R & S & \tilde{\Gamma}_{33} & 0 & 0 & 0 & 0 \\
D_{i}^{T} P & 0 & 0 & -\varepsilon I & \tau_{m} D_{i}^{T} & \delta D_{i}^{T} & 0 \\
\tau_{m} A_{i} & \tilde{\Gamma}_{25}^{T} & 0 & \tau_{m} D_{i} & -R^{-1} & 0 & 0 \\
\delta A_{i} & \tilde{\Gamma}_{26}^{T} & 0 & \delta D_{i} & 0 & -S^{-1} & 0 \\
E_{i} & \tilde{\Gamma}_{27}^{T} & 0 & 0 & 0 & 0 & \tilde{\Gamma}_{77}
\end{array}\right]
$$

with

$$
\begin{aligned}
& \tilde{\Gamma}_{11}=P A_{i}+A_{i}^{T} P+Q-R \\
& \tilde{\Gamma}_{12}=P B_{i} K_{j} \\
& \tilde{\Gamma}_{25}=\tau_{m}\left(B_{i} K_{j}\right)^{T} \\
& \tilde{\Gamma}_{26}=\delta\left(B_{i} K_{j}\right)^{T} \\
& \tilde{\Gamma}_{27}=\left(E_{i b} K_{j}\right)^{T} \\
& \tilde{\Gamma}_{33}=-Q-R-S \\
& \tilde{\Gamma}_{77}=-\varepsilon^{-1} I .
\end{aligned}
$$

Pre- and postmultiplying both sides of the aforesaid matrix inequality (40) by

$$
\left[\begin{array}{ccccccc}
X & X & X & 0 & 0 & 0 & 0 \\
0 & X & 0 & 0 & 0 & 0 & 0 \\
0 & X & X & 0 & 0 & 0 & 0 \\
0 & 0 & 0 & \varepsilon^{-1} I & 0 & 0 & 0 \\
0 & 0 & 0 & 0 & I & 0 & 0 \\
0 & 0 & 0 & 0 & 0 & I & 0 \\
0 & 0 & 0 & 0 & 0 & 0 & I
\end{array}\right]
$$

and its transpose, respectively, and setting $Y_{j}=K_{j} X, \tilde{Q}=$ $X Q X, \tilde{\varepsilon}=\varepsilon^{-1}$ yields (24). This completes the proof.

\section{REFERENCES}

[1] F.-L. Lian, J. Moyne, and D. Tilbury, "Modelling and optimal controller design of networked control systems," Int. J. Control, vol. 76, no. 6, pp. 591-606, Apr. 2003.

[2] F.-L. Lian, J. Moyne, and D. Tilbury, "Network design consideration for distributed control systems," IEEE Trans. Control Syst. Technol., vol. 10, no. 2, pp. 297-307, Mar. 2002.

[3] R. M. Murray, Control in an Information Rich World: Report of the Panel on Future Directions in Control, Dynamics and Systems. Philadelphia, PA: SIAM, 2003.

[4] Y. Tipsuwan and M.-Y. Chow, "Control methodologies in networked control systems," Control Eng. Pract., vol. 11, no. 10, pp. 1099-1111, Oct. 2003.

[5] D. Yue, Q.-L. Han, and C. Peng, "State feedback controller design of networked control systems," IEEE Trans. Circuits Syst. II-Exp. Briefs, vol. 51, no. 11, pp. 640-644, Nov. 2004.

[6] D. Yue, Q.-L. Han, and J. Lam, "Network-based robust $H_{\infty}$ control of systems with uncertainty," Automatica, vol. 41, no. 6, pp. 999-1007, Jun. 2005.

[7] W. Zhang, M. Branicky, and S. Phillips, "Stability of networked control systems," IEEE Control Syst. Mag., vol. 21, no. 1, pp. 84-99, Feb. 2001.

[8] Y. Halevi and A. Ray, "Integrated communication and control systems: Part I-Analysis," ASME J. Dyn. Syst. Meas. Control, vol. 110, no. 4, pp. 367-373, Dec. 1988.

[9] J. Nilsson, "Real-time control systems with delays," Ph.D. dissertation, Lund Inst. Technol., Lund, Sweden, 1998.

[10] G. C. Walsh, H. Ye, and L. G. Bushnell, "Stability analysis of networked control systems," IEEE Trans. Control Syst. Technol., vol. 10, no. 3, pp. 438-446, May 2002.

[11] F. Gökas, "Distributed control of systems over communication networks," Ph.D. dissertation, Univ. Pennsylvania, Philadelphia, 2000.

[12] B. Chen and X. Liu, "Delay-dependent robust $H_{\infty}$ control for T-S fuzzy systems with time delays," IEEE Trans. Fuzzy Syst., vol. 13, no. 4 pp. 544-556, Aug. 2005.

[13] C.-L. Chen, G. Feng, and X.-P. Guan, "Delay-dependent stability analysis and controller synthesis for discrete-time T-S fuzzy systems with time delays," IEEE Trans. Fuzzy Syst., vol. 13, no. 5, pp. 630-643, Oct. 2005.

[14] P. Seiler, "Coordinated control of unmanned aerial vehicles," Ph.D. dissertation, Univ. California, Berkeley, 2001.

[15] Q. Ling and M. D. Lemmon, "Optimal dropout compensation in networked control systems," in Proc. 42nd IEEE Conf. Decis. Control (CDC 2003), Hawaii Island, HI, Dec., pp. 670-675.

[16] G. C. Walsh, O. Beldiman, and L. G. Bushnell, "Asymptotic behavior of nonlinear networked control systems," IEEE Trans. Autom. Control, vol. 46, no. 7, pp. 1093-1097, Jul. 2001.

[17] D. Nešic and A. R. Teel, "Input-to-state stability of networked control systems," Automatica, vol. 40, no. 12, pp. 2121-2128, Dec. 2004.

[18] T. Takagi and M. Sugeno, "Fuzzy identification of systems and its applications to modeling and control," IEEE Trans. Syst., Man, Cybern., vol. SMC-15, no. 1, pp. 116-132, Jan./Feb. 1985.

[19] H. Wang, K. Tanaka, and M. Griffin, "Parallel distributed compensation of nonlinear systems by Takagi and Sugeno fuzzy model," in Proc. IEEE Int. Conf. Fuzzy Syst. (FUZZ-IEEE 1995), pp. 531-538.

[20] H. Wang, K. Tanaka, and M. Griffin, "An approach to fuzzy control of nonlinear systems: Stability and design issues," IEEE Trans. Fuzzy Syst., vol. 4, no. 1, pp. 14-23, Feb. 1996.

[21] Q.-L. Han, "Absolute stability of time-delay systems with sector-bounded nonlinearity," Automatica, vol. 41, no. 12, pp. 2171-2176, Dec. 2005.

[22] Q.-L. Han, "On robust stability of neutral systems with time-varying discrete delay and norm-bounded uncertainty," Automatica, vol. 40, no. 6, pp. 1087-1092, Jun. 2004.

[23] Q.-L. Han, "Robust stability for a class of linear systems with time-varying delay and nonlinear perturbations," Comput. Math. Appl., vol. 47, no. 8-9, pp. 1201-1209, Apr./May 2004.

[24] W. Tang, G. Chen, and R. Lu, "A modified fuzzy PI controller for a flexible-joint robot arm with uncertainties," Fuzzy Sets Syst., vol. 118, no. 1, pp. 109-119, Feb. 2001. 
[25] S. P. Banks, E. Gurkan, and I. Erkmen, "Stable controller design for T-S fuzzy systems based on Lie algebras," Fuzzy Sets Syst., vol. 156, no. 2, pp. 226-248, Dec. 2005.

[26] X. Mao, N. Koroleva, and A. Rodkina, "Robust stability of uncertain stochastic differential delay equations," Syst. Control Lett., vol. 35, no. 5, pp. 325-336, Dec. 1998.

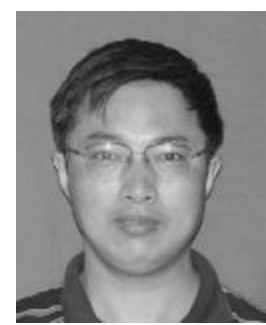

Xiefu Jiang was born in Jiangsu, China, in 1963. $\mathrm{He}$ received the B.S. degree in mathematics from Suzhou University, Jiangsu, in 1985, and the M.S. and Ph.D. degrees in control science and engineering from Southeast University, Nanjing, China, in 1999 and 2002, respectively.

From January 2002 to January 2004, he was a Postdoctoral Researcher Fellow at Tsinghua University, Beijing, China. From February 2004 to February 2007, he was a Postdoctoral Researcher Fellow at Central Queensland University, Rockhampton, QLD, Australia. Since March 2007, he has been a Professor in the School of Automation, Hangzhou Dianzi University, Hangzhou, China. His current research interests include time-delay systems, robust control, fuzzy control, and networked control systems.

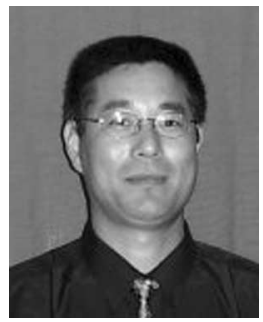

Qing-Long Han received the B.Sc. degree in mathematics from Shandong Normal University, Jinan, China, in 1983, and the M.Eng. and Ph.D. degrees in information science (electrical engineering) from East China University of Science and Technology, Shanghai, China, in 1992 and 1997, respectively.

From September 1997 to December 1998, he was a Postdoctoral Researcher Fellow at LAII-ESIP, Université de Poitiers, Poitiers, France. From January 1999 to August 2001, he was a Research Assistant Professor in the Department of Mechanical and Industrial Engineering, Southern Illinois University, Edwardsville. In September 2001, he joined the Central Queensland University, Rockampton, QLD, Australia, where he is currently a Professor in the School of Computing Sciences, and an Associate Dean (research and innovation) with the Faculty of Business and Informatics. He has been a Visiting Professor at LAII-ESIP, a Chair Professor at Hangzhou Dianzi University, Hangzhou, China, and a Guest Professor at three Chinese universities. His current research interests include time-delay systems, robust control, networked control systems, neural networks, complex systems, and software development processes. He is the author or coauthor of more than 140 refereed papers in technical journals and conference proceedings.

Prof. Han has served as an Associate Editor for the Dynamics of Continuous, Discrete, and Impulsive Systems-Series B: Applications and Algorithms (from 2004 to present), a Guest Editor for the Special Issue on Time-Delay Systems in Dynamics of Continuous, Discrete, and Impulsive Systems-Series B: Applications and Algorithms (from 2006 to present), and a Guest Editor for the Special Issue on Time-Delay Systems in the Asian Journal of Control (from 2004 to 2006). 